Doi: HTTPS://DOI.ORG/10.23910/IJBSM/2018.9.1.3C0530

\title{
Constraint in Adoption of Neem Coated Urea (NCU) in Madhya Pradesh
}

\author{
R. S. Chouhan ${ }^{1^{*}}$, H. K. Niranjan ${ }^{2}$, H. O. Sharma ${ }^{3}$, D. Rathi ${ }^{4}$ and H. S. Kurmi ${ }^{5}$
}

Agro-Economic Research Centre, Jawaharlal Nehru Krishi Vishwa Vidyalaya, Jabalpur, Madhya Pradesh (482 004), India

\section{Corresponding Author}

R. S. Chouhan

e-mail: rsc.aerc@gmail.com

\author{
Article History \\ Article ID: $3 \mathrm{C0530}$ \\ Received in $18^{\text {th }}$ October, 2017 \\ Received in revised form $21^{\text {st }}$ December, 2017 \\ Accepted in final form $28^{\text {th }}$ January, 2018
}

\begin{abstract}
The study comprising of 400 respondents of two major kharif crops i.e. paddy and soybean of Madhya Pradesh to analyze the adoption behavior, constraints in adoption of NCU fertilizers among selected farmers. More than $60 \%$ farmers were found to be aware to NCU and majority of them reported that the main source of awareness was officials of Agriculture Department in Madhya Pradesh. Lack of knowledge about uses of NCU, difficulty in calculating the RDF from different brand of fertilizer available in the market, lake of knowledge about method of application of fertilizer, and lack of awareness about fertilizer use in crop husbandry are the major constraints reported by majority in the area under study. Awareness amongst farmers regarding integrated nutrients management with NCU is required to be created. Packaging/minikit of fertilizer for an acre should be done in such a way so that one bag of fertilizer will serve the purpose of applying recommended doses of fertilizers for different crops as per Agro-Climatic Zones of the state. The farmers are not able to calculate desired nutrients to be applied from the various brands of fertilizers available in the market having different proportion of nutrients. Introduction of national Gateway with respect to e-marketing of seed fertilizer and other inputs at the door step of the farmers to assure quality at reasonable price and timely delivery at desired place.
\end{abstract}

Keywords: Constraint, Adoption, NCU, Madhya Pradesh

\section{Introduction}

The role of chemical fertilizers for increased agricultural production, in particular in developing countries, is well established. Some argue that fertilizer was as important as seed in the Green Revolution (Tomich et al., 1995), contributing as much as $50 \%$ of the yield growth in Asia (Hopper, 1993 and FAO, 1998). Others have found that one-third of the cereal production worldwide is due to the use of fertilizer and related factors of production (Bumb, 1995).

India is the second largest consumer of fertilizer in the world next to China, while it is the third largest producer of nitrogenous fertilizer in the world after China and USA. In terms of Nutrient-wise also, it stands second in the consumption of nitrogen $(\mathrm{N})$ and phosphorus $(\mathrm{P})$ with the quantity of 16.75 million tonnes and 5.63 million tonnes, respectively. Total consumption of NPK fertilizers in the country in 2013 was 24.48 million tonnes (IFA, 2015). About $80 \%$ of the nitrogen was manufactured within the country, whereas the entire potash requirement and $90 \%$ of the phosphate requirement was met through imports during 2000-01 to 2013-14 (Indian Fertilizer
Scenario, 2013).

Soil fertility is determined by three major elements namely Nitrogen, Phosphorus \& Potassium ( $, P, K$ ) of which nitrogen plays a very important role. Out of 17 nutrients essentially required by crop plants for their normal growth and reproduction, nitrogen $(N)$ is generally required by them in the largest amounts. Urea is one of the most widely used sources of nitrogenous fertiliser in the world. It also has high nitrogen content $(46 \%)$, in comparison to many other popular nitrogenous sources. When applied to soil, urea transformed into ammonical $\left(\mathrm{NH}_{4+}\right)$ form after its hydrolysis and then to nitrite $\left(\mathrm{NO}_{2}\right)$, followed by nitrate $\left(\mathrm{NO}_{3}\right)$ form by the process of nitrification. Use of NCU not only increases the crop yield but at the same time lowers the input cost to farmer. Increasing nitrogen efficiency means reducing ground water and air pollution by nitrates and ammonia, respectively. It also reduces import of precious fertilizers as well as reduces ground and soil pollution. NCU has been demonstrated to improve nitrogen use efficiency and consequently crop yield especially in paddy crop and wheat. Coating with Neem oil also reduces caking 
of urea and chances of powder formation during transportation and handling. Repellent action of Neem oil also protects crops fromr many insects; pest and even rats are also go away due to bitter smell of Neem oil. Neem oil is used to manufacture Neem oil insecticide because it contains Azadirachtin which affects over 600 species of pests including insects, nematodes, fungi and viruses and is completely safe to non-target organisms like beneficial predators, honey bees, pollinators, fish, birds, cattle and human beings. Azadirachtin of Neem oil is a famous natural Anti-fee dent, growth regulator and ovi-positional repellent for insects, as a major active ingredient which make it a perfect alternative to chemical pesticides. There are lot of differences in Neem Coated Urea (NCU) and the Normal Urea (NU). In NCU, a layer of Neem over the plain urea that increases the soil fertility capacity that leads to the higher production of crops. The oil coating of Neem in NCU mixes up slowly with the soil and the crop soaks it according to the need. The unwanted urea washed away with the water or gets diluted in the air as nitrogen. If the farmer uses NU, the maximum unit of the manure is left unused. Keeping this review mind the study analyzes the adoption behavior, constraints in adoption of NCU fertilizers among selected farmers.

\section{Materials and Methods}

The study confined to two major kharif crops i.e. paddy and soybean of Madhya Pradesh in the year 2015-16. A multistage purposive sampling method was used to select the districts, blocks, villages and farm households. At the first stage two districts having highest area under and highest consumption of Urea/NCU have been selected purposively for paddy and soybean. Therefore, Balaghat \& Seoni (paddy) and Khargone and Dhar (soybean) districts have been selected in Madhya
Pradesh. In second stage, two blocks from each selected districts were selected again on the basis of highest area in the paddy $\&$ soybean in these selected districts. From the selected blocks 2 cluster of villages comprising 3-4 villages per cluster have been selected for collection of primary data. A sample of 50 farmer from each block comprising 100 farmers in each district, totalling to 200 farmers to each crop have been selected for the study. Thus, study comprising of 400 respondents of two major kharif crops i.e. Paddy (200) and Soybean (200).

\section{Results and Discussion}

To find out the adoption behavior, constraints in adoption of NCU fertilizers among selected farmers the study deals with the awareness \& sources of information, features differentiate NCU to NU, perception of farmers about NCU \& its benefits and constraints about NCU and its adoption

\subsection{Awareness and sources of information}

The awareness about NCU amongst selected farmers and sources of information that made them aware in the area under study related to the selected crops viz. paddy and soybean were identified across size of holdings are presented in Table 1. It is observed from the data that more than $60 \%$ of small $(79.59 \%)$, medium $(87.13 \%)$ and large $(81.48 \%)$ farmers related to soybean and paddy were found to be aware to NCU in the area under study. The major source of awareness as reported by majority of small (36.33\%), medium (49.50\%) and large (44.44\%) farmers was agricultural officers. The farmers' facilitator, fellow farmers were also found to be the source of information which makes them aware about NCU. The role of other sources viz. print media, wall painting agricultural universities, input shops and suppliers was found to be negligible in creating awareness regarding

Table 1: Awareness and sources of information about NCU among the respondents (\% of farmers)

\begin{tabular}{llccccccccc}
\hline \multirow{2}{*}{ SI. No Sources of Information } & \multicolumn{3}{c}{ Paddy } & \multicolumn{3}{c}{ Soybean } & \multicolumn{3}{c}{ Overall } \\
\cline { 2 - 10 } & & Small & Medium & Large & Small & Medium & Large & Small & Medium & Large \\
\hline \% of farmers aware & 93.28 & 96.43 & 100 & 66.67 & 75.56 & 65.52 & 79.59 & 87.13 & 81.48 \\
\multicolumn{2}{l}{ Sources of awareness } & & & & & & & & & \\
\hline 1 & Agricultural Officer & 63.96 & 75.93 & 76.00 & 21.43 & 26.47 & 26.32 & 36.33 & 49.50 & 44.44 \\
2 & Farmer Facilitator & 3.60 & 1.85 & 8.00 & 66.67 & 55.88 & 73.68 & 24.49 & 19.80 & 29.63 \\
3 & Fellow Farmers & 32.43 & 22.22 & 16.00 & 9.52 & 8.82 & 0.00 & 17.96 & 14.85 & 7.41 \\
4 & Print \& Visual media & 0.00 & 0.00 & 0.00 & 1.19 & 2.94 & 0.00 & 0.41 & 0.99 & 0.00 \\
5 & Wall panting & 0.00 & 0.00 & 0.00 & 0.00 & 0.00 & 0.00 & 0.00 & 0.00 & 0.00 \\
6 & Agricultural University & 0.00 & 0.00 & 0.00 & 0.00 & 0.00 & 0.00 & 0.00 & 0.00 & 0.00 \\
7 & Input shop & 0.00 & 0.00 & 0.00 & 1.19 & 5.88 & 0.00 & 0.41 & 1.98 & 0.00 \\
8 & Company (suppliers) & 0.00 & 0.00 & 0.00 & 0.00 & 0.00 & 0.00 & 0.00 & 0.00 & 0.00 \\
\hline
\end{tabular}


use of NCU in the area under study. These findings were found to be similar with minor variations as regards to respondents related to individual selected crop i.e. paddy and soybean. Although, the role of farmers' facilitator were found to be more as compared to other sources of information as reported by the majority of small (66.67\%), medium (55.88\%) and large (73.68\%) soybean growers in the area under study.

\subsection{Features differentiate NCU to NU}

The percentage of farmers noticed difference in NCU to $\mathrm{NU}$ and features from which they differentiate them are presented in Table 2. It is observed from the data that more than $90 \%$ of small (94.86\%), medium (93.81) and large $(94.74 \%)$ farmers were noticed the difference in

\begin{tabular}{|c|c|c|c|c|c|c|c|c|c|}
\hline \multirow[t]{2}{*}{ Sources of Information } & \multicolumn{3}{|c|}{ Paddy } & \multicolumn{3}{|c|}{ Soybean } & \multicolumn{3}{|c|}{ Overall } \\
\hline & Small & Medium & Large & Small & Medium & Large & Small & Medium & Large \\
\hline $\begin{array}{l}\% \text { of farmers noticed } \\
\text { difference in NCU }\end{array}$ & 93.28 & 96.43 & 100 & 96.43 & 91.18 & 89.47 & 81.63 & 84.16 & 77.78 \\
\hline \multicolumn{10}{|l|}{ Factors } \\
\hline Colour difference & 40.00 & 27.63 & 40.00 & 25.00 & 20.59 & 15.79 & 26.94 & 21.78 & 24.07 \\
\hline Price difference & 09.75 & 06.00 & 09.00 & 29.76 & 29.41 & 10.53 & 14.29 & 12.87 & 7.41 \\
\hline Leaf figure on the bag & 50.25 & 66.37 & 51.00 & 45.24 & 50.00 & 73.68 & 38.37 & 52.48 & 50.00 \\
\hline
\end{tabular}

NCU to NU. The major feature of identification of NCU to NU was found to be leaf figure of Neem on the bag as reported by more than $48 \%$ of small ( $38.37 \%)$, medium $(52.48 \%)$ and large $(50.00 \%)$ farmers. A few of them also reported that they were differentiating NCU to NU by colour difference and price variation. These findings are found to be similar with minor variations in case of respondents related to paddy and soybean.

\subsection{Perception of Farmers about NCU and its Benefits}

The perception of respondents about NCU and NU were taken into consideration on different parameters viz. quality, availability, timely availability, price, benefits in terms of total fertilizer and Urea usage, incidence of pest and diseases attack and accessible of NCU in the market and presented in Table 3. As for as quality of NCU is concerned the majority of farmers reported that the quality of NCU available in the market is of good quality (55.91\%) while, $24.80 \%$ of them reported that its quality was found to be very good. The $49.32 \&$ $17.12 \%$ of paddy and $64.81 \& 35.19 \%$ soybean growers considered NCU as good and very good, respectively. The $96.30 \%$ soybean and $63.01 \%$ paddy growers reported that NCU is available in adequate quantity, while $3.42 \& 3.70 \%$ paddy and soybean growers reported inadequate availability of NCU and $33.56 \%$ (paddy growers) reported no change. At overall level most of the farmers reported that there is adequate (77.17\%) and timely $(84.38 \%)$ availability of NCU with almost same price of urea $(68.90 \%)$ or not very high price $(18.11 \%)$, only $12.99 \%$ farmers reported that price of NCU is high (6.69) and very high (6.30). Most of the farmers reported that there is no change in benefit of NCU in terms of total fertilizer usage as reported by paddy $(80.82 \%)$ and soybean growers (49.07\%), while 34.26 and $12.33 \%$ soybean and paddy growers reported that it has capacity to increase benefits.

The decrease in benefits of NCU in terms of total fertilizer usage was reported by $16.67 \& 6.85 \%$ soybean and paddy growers, respectively. At overall level it was found to be reported by farmers that it has been increased (21.65\%), decreased (11.02\%) and no change (67.32\%). The most of the respondents $(79.30 \%)$ reported that there has been no change in benefits of NCU in term of Urea usage at overall level and among the crops also. The majority of respondents also reported that there was no change in pest and disease infestation after use of NCU in cultivation of crops, while $31.83 \%$ reported that it was decreased. The majority of farmers also reported that $\mathrm{NCU}$ is easily accessible in the market, its accessibility was found to be just similar to NU in the market

\subsection{Constraints and Suggestions about NCU and its} Adoption

The major constraints faced by the farmers in adoption of NCU in cultivation of paddy and soybean were also identified and presented in Table 4. All the farmers in the area under study reported that they did not have knowledge about usage of NCU for other than crop production purposes i.e. silage making, mixed with weedicide and fisheries feed preparation.

The difficulty to calculate the recommended doses of nutrients from the different brands of fertilizers available in the market was reported by $74 \%$ of respondents, lack of technical know how about usage of NCU in split doses and method of application (51\%), conditional tagging of other inputs in buying of fertilizers 


\begin{tabular}{|c|c|c|c|c|c|c|c|c|c|c|c|}
\hline \multicolumn{7}{|c|}{ Table 3: Perception about NCU versus Normal Urea } & \multirow{2}{*}{\multicolumn{5}{|c|}{$\begin{array}{l}\text { Table 4: Major constraints faced by the respondents in } \\
\text { adoption of NCU fertilizer ( } \% \text { of farmers) }\end{array}$}} \\
\hline \multirow[t]{3}{*}{ SI. No } & \multirow{2}{*}{\multicolumn{2}{|c|}{$\begin{array}{l}\text { Paddy } \\
(n=146)\end{array}$}} & \multirow{2}{*}{\multicolumn{2}{|c|}{$\begin{array}{l}\text { Soybean } \\
(n=108)\end{array}$}} & \multirow{2}{*}{\multicolumn{2}{|c|}{$\begin{array}{c}\text { Total } \\
(n=254)\end{array}$}} & & & & & \\
\hline & & & & & & & SI. No. & Constraints & Paddy & Soybean & Overall \\
\hline & No & $\%$ & No & $\%$ & No & $\%$ & 1. & Lack of awareness & 26 & 46 & 36 \\
\hline \multicolumn{7}{|c|}{ Neem Coated Urea quality } & 2. & Lack of technical know & 28 & 74 & 51 \\
\hline Very good & 25 & 17.12 & 38 & 35.19 & 63 & 24.80 & & how about usage of & & & \\
\hline Good & 72 & 49.32 & 70 & 64.81 & 142 & 55.91 & & NCU in split doses and & & & \\
\hline Bad & 48 & 32.88 & 0 & 0 & 48 & 18.90 & & metnod oт арріссапо & & & \\
\hline & 1 & 0.68 & 0 & 0 & 1 & 0.39 & 3. & about usage of NCU & 100 & 100 & 100 \\
\hline \multicolumn{7}{|c|}{ Neem Coated Urea availability } & & for other than crop & & & \\
\hline Adequate & 92 & 63.01 & 104 & 96.3 & 196 & 77.17 & & production purposes & & & \\
\hline Inadequate & 5 & 3.42 & 4 & 3.7 & 9 & 3.54 & & i.e. silage making, & & & \\
\hline No change & 49 & 33.56 & 0 & 0 & 49 & 19.29 & & mixed with weedi- & & & \\
\hline \multicolumn{7}{|c|}{ Timely availability of neem coated urea } & & cide and fisheries feed & & & \\
\hline Yes & 120 & 82.19 & 94 & 87.04 & 214 & 84.25 & 4. & Lack of capital & 18 & 13 & 16 \\
\hline & 26 & 17.81 & 14 & 12.96 & 40 & 15.75 & 5. & High cost of fertilizer & 21 & 16 & 19 \\
\hline \multicolumn{7}{|c|}{ Neem coated urea price } & 6. & Difficulty to calculate & 71.5 & 77 & 74 \\
\hline Very high & 0 & 0.00 & 16 & 14.81 & 16 & 6.30 & & the recommended & & & \\
\hline High & 0 & 0.00 & 17 & 15.74 & 17 & 6.69 & & doses of nutrients & & & \\
\hline Not very high & 1 & 0.68 & 45 & 41.67 & 46 & 18.11 & & from the different & & & \\
\hline Same as urea & 145 & 99.32 & 30 & 27.78 & 175 & 68.90 & & brand of fertilizer & & & \\
\hline \multicolumn{7}{|c|}{ Benefits of NCU in terms of total fertilizer usage } & & available in the mar- & & & \\
\hline Increased & 18 & 12.33 & 37 & 34.26 & 55 & 21.65 & & Not available on time & 13 & 19 & 16 \\
\hline Decreased & 10 & 6.85 & 18 & 16.67 & 28 & 11.02 & 1. & & 13 & 19 & 10 \\
\hline No Change & 118 & 80.82 & 53 & 49.07 & 171 & 67.32 & 9 & Conditional tagging of & 39 & 41 & 40 \\
\hline \multicolumn{7}{|c|}{ Benefits of NCU in terms of urea usage } & & other inputs in buy- & & & \\
\hline Increased & 22 & 15.07 & 11 & 10.19 & 33 & 12.99 & & ing of fertilizers from & & & \\
\hline Decreased & 6 & 4.11 & 14 & 12.96 & 20 & 7.87 & & cooperatives & & & \\
\hline No Change & 118 & 80.82 & 83 & 76.85 & 201 & 79.13 & 10. & Inadequate supply & 23 & 26 & 24 \\
\hline
\end{tabular}

Pest and diseases attack

\begin{tabular}{lcccccc}
\hline Increased & 0 & 0.00 & 0 & 0 & 0 & 0.00 \\
Decreased & 76 & 52.05 & 5 & 4.63 & 81 & 31.89 \\
No Change & 70 & 47.95 & 103 & 95.37 & 173 & 68.11
\end{tabular}

NCU is more easily accessible in the market compared to normal Urea

\begin{tabular}{lcccccc}
\hline Yes (reason) & 0 & 0.00 & 37 & 34.26 & 37 & 14.57 \\
No & 146 & 100.00 & 70 & 64.81 & 216 & 85.04 \\
\hline
\end{tabular}

\section{Conclusion}

More and more field demonstrations regarding usage of NCU in cultivation of crops and its usage in other than crop production purposes i.e. silage making, mixed with weedicide and fisheries feed preparation to be conducted in farmers field. Awareness amongst farmers regarding integrated nutrients management with NCU is required to be created. Packaging/minikit of fertilizer for an acre should be done in such a way so that one bag of fertilizer will serve the purpose of applying RDF for different crops as per Agro-Climatic Zones of the state.

from cooperatives (40\%), lack of awareness (36\%) inadequate supply (24\%), high cost of fertilizer (19\%), lack of capital (16\%), not available on time $(16 \%)$ and distant market (11\%) were found to be major constraints in adoption of NCU at over all level.

\section{References}

Bumb, B., 1995. Global Fertiliser Perspective, 1980- 
2000: The Challenges in Structural Transformation, Technical Bulletin T-42. Muscle Shoals, AL: International Fertiliser Development Center.

Hopper, W., 1993. "Indian Agriculture and Fertiliser: An Outsider's Observations", Keynote address to the FAI Seminar on Emerging Scenario in Fertiliser and Agriculture: Global Dimensions, The Fertiliser Association of India, New Delhi.

Indian Fertilizer Scenario, 2014. Department of Fertilizers, Ministry of Chemicals and Fertilizers, Government of India, extracted through http://fert.
nic.in/sites/default/files/Indian\%20Fertilizer\%20 SCENARIO-2014.pdf

International Fertilizer Industry Association (IFA)., 2015. Extracted on 14th December, 2015 from http://ifadata.fertilizer.org/ucResult.aspx?temp $=20151214103823$

Tomich, T., P., Kilby, and B., Johnson., 1995. Transforming Agrarian Economies: Opportunities Seized, Opportunities Missed, Cornell University Press, Ithaca, New York. 